\title{
Uma Introdução à Farmacologia Comportamental
}

\author{
Jan Luiz Leonardi ${ }^{* a, b} \&$ André Amaral Bravin ${ }^{* * c, d}$ \\ ${ }^{\text {a} P o n t i f i ́ c i a ~ U n i v e r s i d a d e ~ C a t o ́ l i c a ~ d e ~ S a ̃ o ~ P a u l o, ~ S a ̃ o ~ P a u l o, ~ B r a s i l, ~}{ }^{b}$ Núcleo Paradigma de Análise do Comportamento, São Paulo, Brasil, \\ ${ }^{\mathrm{c}}$ Universidade Federal de Goiás, Jataí, Brasil \& ${ }^{\mathrm{d}}$ Universidade de Brasília, Brasília, Brasil
}

\begin{abstract}
RESUMO
O presente artigo tem como objetivo introduzir a farmacologia comportamental, enfatizando-a como uma área que demonstra a relevância da complementaridade entre análise do comportamento e fisiologia. Inicialmente, o texto apresenta um breve histórico da disciplina e explora seu preceito fundamental - o de que os efeitos de uma droga constituem-se em variáveis ambientais que exercem diferentes funções de estímulo em contingências respondentes e operantes. Em seguida, alguns experimentos que investigaram os mecanismos comportamentais de ação das drogas são brevemente descritos. Por fim, o artigo aponta que a investigação sobre as relações droga-comportamento realizada pela farmacologia comportamental está de acordo com os pressupostos filosóficos do behaviorismo radical e com a base conceitual e empírica da análise do comportamento.
\end{abstract}

Palavras-chave: farmacologia comportamental; análise do comportamento; drogas.

\begin{abstract}
An Introduction to Behavioral Pharmacology

The aim of this paper is to introduce behavioral pharmacology, emphasizing it as an area that shows the relevance of the complementarity between behavior analysis and physiology. Initially, the text presents a brief history of the subject and explores its fundamental precept - the assertion that the effects of a drug are environmental variables that exert different stimulus functions in respondent and operant contingencies. Afterwards, some experiments which investigated the behavioral mechanisms of drug action are briefly described. Finally, the paper points out that the investigation on drugbehavior relations carried out by behavioral pharmacology is consistent with the philosophical assumptions of radical behaviorism and with the conceptual and empirical basis of behavior analysis.
\end{abstract}

Keywords: behavioral pharmacology; behavior analysis; drugs.

Skinner, ao longo de sua obra (e.g., 1938/1991, 1953/1965, 1974/1976, 1990), defende que a análise do comportamento é uma ciência independente da fisiologia. Neste sentido, o autor aponta que as descobertas fisiológicas não invalidam a proposta e a pertinência de uma ciência que tem por objeto o comportamento em si mesmo, isto é, uma ciência que se ocupa das relações entre respostas e estímulos. De fato, já em seu primeiro livro, Skinner (1938/1991) afirma que

há dois objetos de estudo independentes (comportamento e sistema nervoso) que devem possuir suas próprias técnicas e métodos e produzir seus respectivos dados. Nenhuma quantidade de informação so- bre o segundo irá "explicar" o primeiro ou ordená-lo sem um tratamento analítico direto por parte de uma ciência do comportamento. (p. 423)

Skinner (1953/1965) argumenta que a neurofisiologia não se explica sem se referir às interações do organismo com o ambiente que lhe é externo. Assim, quando concebe a possibilidade de a fisiologia identificar as condições neurais imediatamente antecedentes às instâncias comportamentais, defende que "será descoberto, então, que estes eventos, por sua vez, são antecedidos por outros eventos neurológicos e estes por outros. Esta série nos levará de volta para os eventos fora do sistema nervoso e, finalmente, fora do organismo" (p. 28).

\footnotetext{
* Endereço para correspondência: Jan Luiz Leonardi: janleonardi@ gmail.com

*** Endereço para correspondência: André Amaral Bravin: andrebravin@yahoo.com
} 
As declarações acima indicam que análise do comportamento e neurociência são disciplinas que possuem domínios diferenciados na investigação do comportamento. Reese (1996a, 1996b) defende que as explicações fisiológicas não podem substituir as explicações comportamentais, mas apenas suplementar o conhecimento sobre o comportamento, uma vez que lidam com os fenômenos que ocorrem dentro do organismo enquanto ele se comporta. Neste sentido, Skinner (1974/1976) afirma que o conteúdo fisiológico não pode ser ignorado no estudo do comportamento, mas pode ser deixado de lado nos programas de pesquisa da análise do comportamento, na medida em que essas ciências se ocupam de partes diferentes do fenômeno comportamental (para uma elucidativa revisão sobre a posição de Skinner sobre o papel da biologia na explicação do comportamento, ver Morris, Lazo, \& Smith, 2004).

Embora a análise do comportamento seja uma ciência independente, isso não significa que o conhecimento acerca do funcionamento biológico do organismo não deva ser acrescido a ela. Diversos autores (e.g., Corchs, 2010; Donahoe \& Palmer, 1994; Ilardi \& Feldman, 2001; Strumwasser, 1994; Timberlake, Schaal, \& Steinmetz, 2005) defendem explicitamente a complementaridade entre análise do comportamento e neurociência, com base no pressuposto de que o estudo dos processos comportamentais e dos processos cerebrais é fundamental para uma explicação completa do comportamento humano. Quanto a essa possibilidade de integração, Skinner (1974/1976) é enfático:

O fisiólogo do futuro nos dirá tudo que pode ser conhecido acerca do que está ocorrendo no interior do organismo em ação. Sua descrição constituirá um progresso importante em relação a uma análise do comportamento, porque esta é necessariamente "histórica" - isto quer dizer que ela está limitada às relações funcionais que envolvem lacunas temporais. Faz-se hoje algo que virá a afetar amanhã o comportamento de um organismo. Não importa quão claramente se possa estabelecer esse fato, falta uma etapa, e devemos esperar que o fisiólogo a estabeleça. Ele será capaz de mostrar como um organismo é modificado quando é exposto a contingências de reforçamento e porque então o organismo modificado se comporta de maneira diferente, em data possivelmente muito posterior. O que ele descobrir não pode invalidar as leis de uma ciência do comportamento, mas tornará o quadro da ação humana mais completo. (pp. 236-237)
Dentre as possibilidades de complementaridade entre as ciências comportamentais e as ciências biológicas, o campo que tem se mostrado mais fértil é o da farmacologia comportamental (Leslie, 2003).

\section{FARMACOLOGIA COMPORTAMENTAL}

A farmacologia comportamental tem como origem uma série de estudos pioneiros conduzidos na década de 1950 no Laboratório de Psicobiologia da Faculdade de Medicina de Harvard. Estabelecidos nesse laboratório, Peter B. Dews, Roger T. Kelleher e William H. Morse foram os pesquisadores que lançaram a base conceitual e o programa experimental que definiu e dominou a farmacologia comportamental (Barrett, 2002), produção cujo impacto foi tão grande que deu origem a essa nova disciplina e a influencia até hoje (para dados biográficos e bibliográficos sobre esses três autores, ver Barrett, 2006; Branch, 2006b; Marr, 2006; Zeiler, 2006).

A farmacologia comportamental surgiu como uma disciplina científica formal em um contexto histórico revolucionário, tanto para a análise do comportamento quanto para a farmacologia. A década de 1950 é comumente denominada como a "idade de ouro" da psicofarmacologia devido à identificação das primeiras drogas efetivas no tratamento dos chamados "transtornos mentais" que revolucionaram a clínica psiquiátrica. Em um período de apenas dez anos, substâncias como clorpromazina, imipramina e clordiazepóxido foram descobertas e introduzidas no tratamento da esquizofrenia, depressão e ansiedade, respectivamente. Isso motivou a pesquisa e o financiamento de novos agentes farmacológicos e a experimentação com animais de laboratório antes de serem testados em humanos (Barrett, 2002, 2006; Bravin, Pandossio, $\&$ Albuquerque, 2007).

A idade de ouro da psicofarmacologia coincide com importantes avanços na análise do comportamento: em 1953, Skinner (1953/1965) publica o livro Science and Human Behavior, no qual apresenta uma sistematização do conhecimento produzido nas décadas anteriores pela pesquisa experimental do comportamento, analisa sua relevância na compreensão dos fenômenos psicológicos e discute sua possível extensão para as relações sociais humanas; em 1957, publica Schedules of Reinforcement (Ferster \& Skinner, 1957), em que compila mais de setenta mil horas de pesquisa experimental com sujeitos não humanos; neste mesmo ano, publica Verbal Behavior (1957/1992), um livro inteiramente dedicado a um comportamento especifica- 
mente humano, o comportamento verbal; em 1958, ocorre a fundação do Journal of the Experimental Analysis of Behavior, principal veículo de publicação de pesquisa experimental básica em análise do comportamento (Barrett, 2002, 2006; Todorov, 2004).

Ao discorrer sobre o contexto histórico do surgimento da farmacologia comportamental, Barrett (2006) explica que "as excepcionais ferramentas da análise experimental do comportamento combinadas com os empolgantes efeitos das drogas descobertas pela ciência farmacológica forneceram enormes possibilidades para essa disciplina emergente" (p. 361). Vale notar que a emergência dessa nova disciplina já parecia ser antecipada por Ferster e Skinner (1957) em Schedules of Reinforcement. Neste livro, os autores destacam a participação de Peter Dews em experimentos que avaliaram a influência de drogas sobre o comportamento de animais expostos a diferentes esquemas de reforçamento e agradecem a J. Lawrence Pool por ter lesionado estruturas cerebrais de pombos para o estudo de seu padrão de respostas, explicitando a possibilidade de investigação comportamental de variáveis biológicas.

Uma das primeiras pesquisas em análise do comportamento envolvendo drogas foi o trabalho de Heron e Skinner (1937), em que foi observado o efeito da cafeína e da benzedrina sobre respostas de pressão à barra de ratos em FI 4 min. e em extinção. Poling (2000) lembra que essa pesquisa não despertou interesse na comunidade científica e que mais de uma década se passou até que a farmacologia comportamental se estabelecesse como uma disciplina formal. A despeito do estudo supracitado, o trabalho considerado por muitos autores (e.g., Barrett, 2006; Blackman \& Pellon, 1993; Branch, 2006a) como o marco inicial da farmacologia comportamental foi o estudo de Dews (1955), que demonstrou que variáveis ambientais podem modificar a maneira pela qual as drogas afetam o comportamento. Dews treinou pombos privados de alimento a bicarem um disco em FR 50 (que gera alta taxa de respostas) e em FI 15 min. (que produz baixa taxa de respostas). Após atingir desempenho estável nesses esquemas, foram realizadas sessões de teste nas quais diferentes doses de pentobarbital e salina (substância inócua) foram administradas nos sujeitos. Os resultados evidenciaram mudanças no responder dos pombos em comparação com o desempenho estável demonstrado na linha de base após a administração de pentobarbital, em especial nas doses de $1.0 \mathrm{mg} / \mathrm{kg}$ e $2.0 \mathrm{mg} / \mathrm{kg}$. Sob efeito dessa doses, a taxa de respostas no esquema FR aumentou, enquanto que a taxa em FI diminuiu, demonstrando que uma droga tradicionalmente classificada como "depressora do sistema nervoso central" pode influenciar tanto o aumento quanto a diminuição da taxa de respostas a depender do esquema de reforçamento em vigor. Barrett (2002, 2006) explica que o estudo de Dews demarcou o escopo das pesquisas em farmacologia comportamental pelas décadas seguintes e que, ao incorporar o controle experimental do comportamento na avaliação dos efeitos das drogas, Dews forneceu à farmacologia comportamental a precisão e o rigor necessários para seu estabelecimento.

Em 1968, a definição e os objetivos da farmacologia comportamental foram explicitamente propostos por Travis Thompson e Charles Schuster em Behavioral Pharmacology, o primeiro livro-texto a sistematizar os conhecimentos produzidos até então na área. Segundo esses autores, a farmacologia comportamental é

um ramo das ciências biológicas que utiliza as ferramentas e conceitos da psicologia experimental e da farmacologia para explorar a ação comportamental das drogas. As ferramentas da psicologia experimental podem ser, para o presente propósito, caracterizadas pela frase "técnicas de controle comportamental". As principais "ferramentas" da farmacologia são as drogas. Portanto, quando alguém estiver interessado no controle comportamental para delinear a ação de uma droga ou estiver utilizando drogas para analisar processos comportamentais, a análise repousa no domínio da farmacologia comportamental. (Thompson \& Schuster, 1968, pp. 1-2)

\section{Na mesma linha, Skinner (1969) afirma que}

As manipulações fisiológicas serão simplesmente acrescentadas ao arsenal do cientista do comportamento. A farmacologia já prenunciou essa situação. Uma droga muda um organismo de maneira tal que ele se comporta diferentemente. Poderíamos produzir a mesma mudança manipulando variáveis ambientais típicas, mas agora a droga permite que evitemos tal manipulação. (p. 283)

As descobertas empíricas da farmacologia comportamental explicitam que os efeitos de qualquer droga dependem tanto das características do comportamento e de seus determinantes ambientais quanto das propriedades químicas da droga. Por isso, Blackman e Pellon (1993) defendem que "uma farmacologia comportamental científica, efetiva e sensível, deve incluir princípios comportamentais assim como princípios farmacológicos, e deve analisar as interações dinâmicas entre variáveis comportamentais e farmacológicas” (p. 21). 
A disciplina elenca como objeto de estudo as relações droga-comportamento, empregando o referencial filosófico, conceitual e metodológico da análise do comportamento. Ao conceber as drogas como eventos constitutivos do ambiente, a farmacologia comportamental assume que elas podem exercer diferentes papéis enquanto variáveis de controle do comportamento em contingências respondentes e em contingências operantes. Isto é o que tem sido descrito pela literatura como mecanismos comportamentais de ação das drogas (Branch, 2006a; Bravin, Pandossio, \& Albuquerque, 2007; Lejuez, Schaal, \& O'Donnell, 1998; Leslie, 2003; Poling, Byrne, \& Morgan, 2000).

\section{Mecanismos comportamentais de ação das drogas}

Antes de apresentar os mecanismos comportamentais de ação das drogas propriamente ditos, é importante apontar que a maior parte dos estudos em farmacologia comportamental utiliza o comportamento operante sob controle de esquemas de reforçamento intermitente para avaliar os efeitos de diferentes substâncias, empregando pombos, ratos e macacos como sujeitos na maioria das vezes. Inúmeras pesquisas (conforme Ferster \& Skinner, 1957) evidenciam que os procedimentos de reforçamento intermitente têm grande utilidade por gerar desempenhos estáveis e previsíveis: um comportamento operante, ao atingir uma taxa de respostas estável em um esquema intermitente, não sofrerá mudanças significativas se as condições de reforçamento não forem modificadas. Por conseguinte, a taxa de respostas mantida por um esquema de reforçamento intermitente pode ser empregada como linha de base para a investigação dos efeitos das drogas sobre um desempenho operante (Catania, 1999; Leslie, 2003). Leslie (2003) explica que a estabilidade da linha de base produzida por esquemas de reforçamento intermitente permite a investigação de aspectos fundamentais na caracterização dos efeitos das drogas, como: (a) monitorar o tempo do efeito comportamental de uma droga; (b) estabelecer a curva dose-efeito; (c) identificar a via de administração mais efetiva para provocar mudanças comportamentais; (d) comparar os efeitos comportamentais de novas substâncias com drogas já utilizadas clinicamente; (e) classificar as drogas de acordo com seus efeitos comportamentais em um ambiente controlado; (f) estabelecer se uma classe de drogas com efeitos clínicos similares tem os mesmos efeitos em procedimentos de condicionamento operante.

No contexto do comportamento respondente, a droga tem função de estímulo incondicional que elicia respostas incondicionais - seus efeitos no organismo. Entre esses efeitos encontram-se respostas compensatórias, pois, diante do distúrbio fisiológico produzido pela droga, o organismo reage com processos regulatórios, opostos aos iniciais, que visam restabelecer o equilíbrio fisiológico anterior (homeostasia). Embora a droga atue como um estímulo incondicional, outras variáveis ambientais também afetam seus efeitos comportamentais (Benvenuti, 2004, 2007; Poling, Byrne \& Morgan, 2000). O ambiente, ao preceder sistematicamente a presença da droga no organismo, exerce função de estímulo condicional e passa a eliciar os processos regulatórios anteriormente eliciados pela ação da droga, o que foi demonstrado em diversos trabalhos experimentais (e.g., Lê, Poulos, \& Cappel, 1979; Siegel, 1975; Siegel, Hinson, Krank, \& McCully, 1982). Desta forma, quantidades cada vez maiores da droga são necessárias para que os efeitos iniciais sejam produzidos no organismo, levando ao fenômeno conhecido como tolerância. Posteriormente a essa história, se a substância for consumida em um ambiente bastante diferente do usual (i.e., na ausência dos estímulos condicionais que eliciam as respostas compensatórias), o organismo pode entrar em colapso, pois não está preparado para receber aquela quantidade da droga, o que é conhecido na literatura por overdose (Siegel, 2001). Além disso, o ambiente (estímulos condicionais) no qual a droga foi utilizada pode eliciar os processos regulatórios (respostas condicionais) mesmo na ausência da substância, produzindo o fenômeno denominado síndrome de abstinência (Benvenuti, 2007; Macrae, Scoles, \& Siegel, 1987).

No paradigma do comportamento operante, as drogas podem exercer diferentes funções comportamentais, como estímulo reforçador, estímulo discriminativo, operação motivadora ou, ainda, interferir em desempenhos comportamentais estáveis (Bravin, Pandossio, \& Albuquerque, 2007; Lejuez, Schaal, \& O’Donnell, 1998; Leslie, 2003; Poling, Byrne, \& Morgan, 2000). Uma propriedade das drogas é a capacidade de funcionarem como estímulos reforçadores positivos e negativos (Schuster, 1976). Experimentos envolvendo diferentes drogas foram realizados com animais de laboratório e com humanos, nos quais mais de 100 compostos foram testados. Em geral, há uma forte correspondência entre não humanos e humanos no que diz respeito à função reforçadora de diversas substâncias, tais como estimulantes, opióides, barbitúricos, benzodiazepínicos e outros sedativos/hipnóticos (Lejuez, Schaal, \& O’Donnell, 1998; Poling, Byrne, \& Morgan, 2000). Para estabelecer o potencial reforça- 
dor de uma droga, são comparados arranjos experimentais que estabelecem um padrão estável de respostas mantidas pela administração da droga com aqueles que empregam água ou alimento como reforçador. Schuster (1976), por exemplo, cita um experimento conduzido em seu laboratório em que o responder de macacos em FR 20 era mantido por diferentes doses de codeína ou metadona (agonistas opióides). Outro exemplo é o estudo de Galuska e Woods (2005), no qual a resposta de pressão à barra de macacos foi modelada utilizando como consequência a administração de cocaína com atraso não sinalizado.

Experimentos desse tipo permitem investigar também a função de reforçador negativo das drogas. Por exemplo, Hoffmeister e Wuttke (1976) delinearam um experimento para avaliar o potencial reforçador negativo de diversas substâncias, entre as quais dietilamida de ácido lisérgico (LSD), nalorfina, clorpromazina, imipramina e pentobarbital. Inicialmente, macacos foram submetidos a um esquema de reforçamento negativo cuja consequência era a retirada de choque e, posteriormente, a um esquema idêntico cuja consequência era a interrupção da administração de diferentes doses das drogas referidas acima. $\mathrm{O}$ experimento mostrou que os animais pressionavam a barra para interromper a infusão da droga em uma proporção dose-dependente (i.e., quanto maior era a dose da substância, mais os animais pressionavam a barra que produzia a fuga), resultado igual a quando o estímulo em questão era o choque (i.e., quanto maior era a amperagem, maior era a taxa de respostas). Além disso, a pesquisa de Hoffmeister e Wuttke (1976) demonstrou também que os sujeitos emitiram respostas que eliminavam os estímulos que antecediam a infusão de algumas das drogas estudadas da mesma forma que o faziam com estímulos emparelhados com choque. Nalorfina, clorpromazina e LSD funcionaram como reforçadores negativos, enquanto imipramina e pentobarbital produziram pouca ou nenhuma resposta de esquiva.

Além de exercer as já citadas funções de reforçador positivo e negativo, drogas podem ter função de estímulo reforçador condicionado quando são pareadas com outros reforçadores. Em uma pesquisa com humanos, Johanson, Mattox e Schuster (1995) avaliaram em que medida substâncias comportamentalmente inócuas eram preferidas após uma história de contingenciamento com outros reforçadores. A ingestão de um dentre dois tipos de placebo (com rótulos e cores distintas) era emparelhado com uma contingência de maior ganho monetário ao passo que o outro com uma de menor ganho monetário. A maioria dos participantes demonstrou preferência ao placebo correlacionado com a contingência de maior ganho monetário, revelando que a substância inócua adquiriu valor de reforço condicionado. Estendendo essa descoberta, Alessi, Roll, Reilly e Johanson (2002) substituíram um dos placebos por diazepam, fármaco geralmente preterido por humanos nos experimentos. Na primeira fase, os pesquisadores avaliaram se a droga era de fato evitada pelos participantes da pesquisa quando comparada ao placebo em uma escolha livre de autoadministração, o que ocorreu para cinco dos seis participantes. Na segunda etapa, eles recebiam novamente droga ou placebo (em outras cápsulas, para evitar efeito de aprendizagem com estímulos exteroceptivos) e eram submetidos a sessões de jogos de computador em que recebiam maiores quantias de dinheiro sob efeito do diazepam independentemente do comportamento emitido. Depois de quatro sessões consecutivas, os participantes foram submetidos a uma nova condição de escolha, onde passaram a autoadministrar o diazepam, que foi preterido na primeira etapa do experimento. Em suma, a escolha foi invertida quando a droga foi pareada com outro estímulo reforçador.

Esses achados possuem uma clara relação com o contexto de aplicação, na medida em que muitas drogas são autoadministradas por seu valor reforçador condicionado a reforçadores disponibilizados por determinados grupos sociais. Isto pode propiciar a ingestão de substâncias preteridas em um primeiro momento de forma a instalar e manter o comportamento de uso de substâncias que, com o tempo, pode passar a ficar também sob controle dos efeitos fisiológicos inerentes a elas.

A estimulação encoberta produzida pela administração de uma droga pode exercer função de estímulo discriminativo ( $\mathrm{Sd}$ ), desde que duas condições sejam obedecidas: (a) a droga deve produzir efeitos detectáveis pelo organismo e (b) o organismo deve passar por uma história de reforçamento diferencial na presença e na ausência da droga (Poling, Byrne, \& Morgan, 2000). Assim, em um experimento típico, uma resposta é reforçada quando determinada droga é administrada e não é reforçada quando outra droga ou uma substância inócua é aplicada. Kamien, Bickel, Hughes, Higgins e Smith (1993) conduziram uma revisão sobre as pesquisas que investigaram o papel discriminativo que as drogas podem exercer em humanos e não humanos, concluindo que seus efeitos são parecidos entre as espécies para uma grande variedade de agentes, como anfetaminas, opióides, benzodiazepínicos, cafeína, etanol, THC e nicotina. 
O comportamento de organismos que passam por um treino discriminativo com uma determinada droga pode se generalizar para outras doses da mesma droga ou para diferentes substâncias com propriedades farmacológicas em comum (e.g., drogas estimulantes, tais como metilfenidato, cocaína e anfetamina). Um exemplo de generalização envolvendo diferentes doses de uma mesma droga é o trabalho de Chait, Uhlenhuth e Johanson (1984). Inicialmente, os participantes ingeriram d-anfetamina ou placebo por dois dias (período de amostragem), substâncias nomeadas como droga $A$ ou droga $B$. Em seguida, os participantes passaram por seis dias de treino, nos quais receberam aleatoriamente a $\operatorname{droga} A$ em três desses dias e a $\operatorname{droga} B$ nos dias restantes. Após deixarem o laboratório, os participantes respondiam a um questionário sobre estados de humor uma, três e seis horas depois de receberem a substância. Ao completarem o último questionário do dia (i.e., seis horas depois de receberem a substância), eles ligavam para os pesquisadores, que reforçavam diferencialmente (com dinheiro no dia seguinte) a discriminação correta da droga. Após o treino e a verificação da aprendizagem discriminativa (acerto na discriminação em cinco das seis tentativas possíveis), os participantes seguiram para a fase de teste, na qual receberam um dos seguintes tratamentos por dez dias (repetido duas vezes para substância): $2.5 \mathrm{mg}, 5 \mathrm{mg}$ e $10 \mathrm{mg}$ de d-anfetamina; $10 \mathrm{mg}$ de diazepam; placebo. Quando os participantes estavam sob a dose teste de $2.5 \mathrm{mg} / \mathrm{kg}$ de anfetamina, o percentual de respostas corretas foi de $10 \%$ do valor obtido na linha de base (idêntico ao placebo), aumentando para $40 \%$ quando esta estava sob efeito de $5 \mathrm{mg} / \mathrm{kg}$, chegando a $100 \%$ (i.e., idêntico aos valores da linha de base) quando estava sob a dose de $10 \mathrm{mg} / \mathrm{kg}$. Os dados permitiram identificar um gradiente de generalização à droga (enquanto $\mathrm{Sd}$ ) dentre as doses exploradas.

Por sua vez, um exemplo de generalização envolvendo diferentes drogas de uma mesma classe é descrita no estudo de Lubinski e Thompson (1987). Os autores tinham como objetivo determinar se um operante discriminado, sob controle de um estímulo discriminativo interoceptivo, se generalizaria a outros estados similares, mas de alguma maneira diferentes aos previamente induzidos. Embora o experimento tenha envolvido um arranjo complexo de contingências, para o presente propósito cabe descrever que pombos eram treinados a bicar um dentre três discos enquanto uma luz azul piscava. Os discos apresentavam as letras $D, S$ ou $N$, cujas bicadas eram reforçadas quando existia uma correspondência entre o efeito fisiológico produzido pela substância e o símbolo em questão. Neste caso, bicadas em $D$ eram reforçadas quando o animal estava sob efeito do pentobarbital (um depressor do sistema nervoso), bicadas em $S$ eram reforçadas quando o animal estava sob efeito de cocaína (um estimulante do sistema nervoso) e bicadas em $N$ eram reforçadas quando o animal estava sob efeito do veículo (salina, substância inerte). O resultados (Experimento 1) revelaram que, após o treino, todos os animais mostraram alto grau de acurácia, apresentando mais de $90 \%$ de correspondências entre o estado fisiológico e a letra do operandum. No Experimento 2, a droga de treino foi substituída por drogas diferentes, mas pertencentes à mesma classe farmacológica; isto é, pentobarbital e cocaína foram respectivamente substituídas por clordiazepóxido e danfetamina (outro depressor e outro estimulante, respectivamente). Os resultados demonstraram que a discriminação interoceptiva se generalizou para o clordiazepóxido e para a d-anfetamina, e que estes produziram efeitos interoceptivos similares (embora não fossem os mesmos), o que foi confirmado pelo percentual de acertos, que variou entre $88 \%$ e $100 \%$. Em resumo, o estudo mostrou que o controle discriminativo das drogas treinadas se estendeu para as novas drogas com alta porcentagem de acerto.

No que concerne à aplicação, a função discriminativa das drogas pode explicar, ao menos parcialmente, a disrupção de um operante quando uma substância deixa de ser administrada. Considere o exemplo hipotético de uma pessoa que tenha aprendido a alimentarse adequadamente sob o efeito psicoestimulante de anorexígenos (e.g., dietilpropiona). Uma vez que tenha ocorrido o treino discriminativo de menor ingestão calórica mediante o efeito da droga (e consequente perda de peso) e de maior ingestão calórica na ausência de sua estimulação (e consequente manutenção ou ganho de peso), a presença da substância no organismo pode passar a controlar o comportamento de escolha por alimentos menos calóricos. A retirada abrupta do medicamento ou a alteração de sua concentração no organismo pode funcionar como o desligamento de uma luz com função de $\mathrm{Sd}$ para respostas de pressão à barra de um rato em uma caixa experimental. Em ambos os casos, ocorre a perda do controle de estímulos, o que acarreta na diminuição da taxa de respostas. Outro exemplo do potencial prático da função discriminativa das drogas foi descrito por Lovibond e Caddy (1970), que argumentam a favor da possibilidade de treinar a discriminação interoceptiva do nível de álcool no sangue para facilitar o controle do consumo de etanol no tratamento de alcoolistas. 
Outra função comportamental que uma droga pode exercer é a de operação motivadora, alterando momentaneamente a eficácia de reforçadores ou punidores e a frequência das classes operantes relacionadas àquelas consequências (conforme Laraway, Snycerski, Michael, \& Poling, 2003; Miguel, 2000). Uma evidência bem estabelecida na literatura é a diminuição da frequência de respostas de buscar alimento produzida pelo consumo de anfetamina (Poling, Byrne, \& Morgan, 2000). Nesta linha de investigação, Northup, Fusilier, Swanson, Roane e Borrero (1997) avaliaram se o metilfenidato poderia ter função de operação motivadora para crianças com diagnóstico de desatenção e hiperatividade. Para isso, os autores compararam diversos métodos de avaliação de preferência por diferentes estímulos reforçadores comumente utilizados na aplicação de procedimentos comportamentais (itens comestíveis, itens tangíveis, atividades, atenção, etc.), obtidos por meio de um procedimento de economia de fichas. Os resultados da pesquisa mostraram que a droga aumentou a frequência de classes de respostas relacionadas a alguns dos estímulos e diminuiu a frequência do responder relacionado a outros estímulos. Esses dados permitem inferir que houve uma alteração momentânea do valor reforçador dos diferentes estímulos utilizados, ou seja, que a droga exerceu função de operação motivadora.

As pesquisas experimentais que analisam as funções comportamentais das drogas - estímulo eliciador, estímulo reforçador, estímulo discriminativo e operação motivadora - são muito importantes na avaliação do potencial de abuso de novas substâncias, no desenvolvimento de tratamentos para dependência química, na compreensão dos mecanismos neurobiológicos subjacentes à ação das substâncias e na ampliação do conhecimento sobre processos comportamentais básicos, entre outros (Branch, 2006a).

\section{CONSIDERAÇÕES FINAIS}

O presente trabalho apresentou a área da farmacologia comportamental, apontando que a união da análise do comportamento com as ciências biológicas talvez seja inevitável, sobretudo em um conhecimento interdisciplinar como o estudo das relações drogacomportamento, desde que tal união não obscureça a filosofia behaviorista radical. Como atesta Lattal (2005),

trazer a pesquisa e a teoria de outras disciplinas é, porém, tanto inevitável quanto pode ser altamente útil em expandir o âmbito da análise do comportamento. Dependendo de como a mistura é feita, a vi- são de mundo não precisa ser necessariamente comprometida. (p. 23)

Aludindo à farmacologia comportamental, este autor acrescenta ainda que "o estudo de ações de drogas poderia expandir o entendimento dos mecanismos tanto das drogas quanto do comportamento" (p. 23). A proposição do autor é que o estudo das drogas contribui para o entendimento do estudo do comportamento e vice-versa, o que configura certamente uma postura diferente daqueles que "simplesmente usam os métodos da análise do comportamento como uma tecnologia para estudar a ação de drogas" (p. 23).

De fato, estudos dessa natureza geralmente encontram-se vinculados a outra tradição epistemológica, cujo pressuposto é de que as drogas são condições necessárias e suficientes para a mudança observada no comportamento. A psicofarmacologia, por exemplo, pressupõe que o efeito comportamental de uma droga pode ser explicado quase que exclusivamente por sua ligação química a determinados receptores. Certamente, nenhum analista do comportamento seria suficientemente ingênuo para descartar o conhecimento acerca do funcionamento neurobiológico das drogas. Entretanto, a explicação exclusivamente biológica de um comportamento que é qualitativamente diferente por estar, entre outras variáveis, sob controle do efeito da droga, é o que fere os preceitos comportamentais.

Ao delimitar seu objeto de estudo ao funcionamento neurobiológico das drogas, a psicofarmacologia produz um conhecimento incompleto acerca das relações droga-comportamento. A despeito dessa limitação, o enfoque e o financiamento da pesquisa nas últimas décadas têm sido majoritariamente sobre os mecanismos moleculares da ação das drogas, sejam elas terapêuticas ou não, em detrimento do estudo pela interação entre droga e comportamento (Barrett \& Sanger, 1991). Para o psicólogo e farmacologista DeGrandpre (2006), a relevância das pesquisas em psicofarmacologia é restrita porque estão comprometidas com aquilo que ele denomina de farmacologismo:

O farmacologismo, como o racismo, é um sistema ideológico embasado em uma série de pressupostos que, embora falsos e exagerados, governam um conjunto de percepções, entendimentos e ações. Uma suposição central do farmacologismo é que as potencialidades farmacológicas contidas na estrutura química da droga determinam os efeitos da droga no corpo, no cérebro e no comportamento. (p. 27)

Inversamente, a farmacologia comportamental fornece explicações das relações droga-comportamento 
observadas no laboratório e na vida cotidiana que levam em conta tanto as propriedades farmacológicas das substâncias quanto outras variáveis ambientais. Desta forma, a investigação sobre as relações droga-comportamento está de acordo com os pressupostos filosóficos do behaviorismo radical e da base conceitual e empírica da análise do comportamento, o que torna possível compreender a influência de variáveis biológicas na determinação do comportamento humano sem incorrer em noções mentalistas ou reducionistas.

\section{REFERÊNCIAS}

Alessi, S. M., Roll, J. M., Reilly, M. P., \& Johanson, C. E. (2002). Establishment of a diazepam preference in human volunteers following a differential-conditioning history of placebo versus diazepam choice. Experimental and Clinical Psychopharmacology, 10, 77-83.

Barrett, J. E., \& Sanger, D. J. (1991). Behavioral pharmacology in the era of neuroscience. Journal of the Experimental Analysis of Behavior, 56, 167-169.

Barrett, J. E. (2002). The emergence of behavioral pharmacology. Molecular Interventions, 2, 470-475.

Barrett, J. E. (2006). Behavioral determinants of drug action: The contributions of Peter B. Dews. Journal of the Experimental Analysis of Behavior, 86, 359-370.

Benvenuti, M. F. (2004). Condicionamento respondente: Algumas implicações para o desenvolvimento de tolerância, síndrome de abstinência e overdose. Em C. N. Abreu \& H. J. Guilhardi (Orgs.), Terapia comportamental e cognitivo-comportamental: Práticas clínicas (pp. 186-193). São Paulo: Roca.

Benvenuti, M. F. (2007). Uso de drogas, recaída e o papel do condicionamento respondente: Possibilidades do trabalho do psicólogo em ambiente natural. Em D. R. Zamignani, R. Kovac, \& J. S. Vermes (Orgs.), A clínica de portas abertas: Experiências e fundamentação do acompanhamento terapêutico $e$ da prática clínica em ambiente extraconsultório (pp. 307-327). São Paulo: Paradigma/ESETec.

Blackman, D. E., \& Pellon, R. (1993). The contributions of B. F. Skinner to the interdisciplinary science of behavioural pharmacology. British Journal of Psychology, 84, 1-25.

Branch, M. N. (2006a). How research in behavioral pharmacology informs behavioral science. Journal of the Experimental Analysis of Behavior, 85, 407-423.

Branch, M. N. (2006b). Roger T. Kelleher, behavior analyst. Journal of the Experimental Analysis of Behavior, 86, 371-384.

Bravin, A. A., Pandossio, J. E., \& Albuquerque, A. R. (2007). O que é farmacologia comportamental? Em W. C. M. Pereira da Silva (Org.), Sobre comportamento e cognição: Vol. 20. Reflexões teórico-conceituais e implicações para pesquisa (pp. 123136). Santo André: ESETec.

Catania, A. C. (1999). Aprendizagem: Comportamento, linguagem e cognição (4a ed.). (D. G. Souza, Trad.). Porto Alegre: Artmed.

Chait, L. D., Uhlenhuth, E. H., \& Johanson, C. E. (1984). An experimental paradigm for studying the discriminative stimulus properties of drugs in humans. Psychopharmacology, 82, 272274.
Corchs, F. (2010). É possível ser um psiquiatra behaviorista radical? Primeiras reflexões. Perspectivas em Análise do Comportamento, 1, 55-66.

DeGrandpre, R. (2006). The cult of pharmacology: How America became the world's most troubled drug culture. Durham, NC: Duke University Press.

Dews, P. B. (1955). Studies on behavior I. Differential sensitivity to pentobarbital of pecking performance in pigeons depending on the schedule of reward. Journal of Pharmacology and Experimental Therapeutics, 113, 393-401.

Donahoe, J. W., \& Palmer, D. C. (1994). Learning and complex behavior. Boston, MA: Allyn \& Bacon.

Ferster, C. B., \& Skinner, B. F. (1957). Schedules of reinforcement. Acton, MA: Copley.

Galuska, C. M., \& Woods, J. H. (2005). Acquisition of cocaine self-administration with unsignaled delayed reinforcement in rhesus monkeys. Journal of the Experimental Analysis of Behavior, 84, 269-280.

Heron, W. T., \& Skinner, B. F. (1937). Effects of caffeine and benzedrine upon conditioning and extinction. The Psychological Record, 1, 340-346.

Hoffmeister, F., \& Wuttke, W. (1976). Psychotropic drugs as negative reinforcers. Pharmacological Reviews, 27, 419-428.

Ilardi, S. S., \& Feldman, D. (2001). Toward rapprochement: Comments on the role of biological science within cognitive neuroscience and radical behaviorism. Journal of Clinical Psychology, 57, 1121-1124.

Johanson, C. E., Mattox, A., \& Schuster, C. R. (1995). Conditioned reinforcing effects of capsules associated with high versus low monetary payoff. Psychopharmacology, 120, 42-48.

Kamien, J. B., Bickel, W. K., Hughes, J. R., Higgins, S. T., \& Smith, B. J. (1993). Drug discrimination by humans compared to nonhumans: Current status and future directions. Psychopharmacology, 111, 259-270.

Kelleher, R. T., \& Morse, W. H. (1964). Escape behavior and punished behavior. Federation Proceedings, 23, 808-817.

Laraway, S., Snycerski, S., Michael, J., \& Poling, A. (2003). Motivative operations and terms to describe them: Some further refinements. Journal of Applied Behavior Analysis, 36, 407-414.

Lattal, K. A. (2005). Ciência, tecnologia e análise do comportamento. Em J. Abreu-Rodrigues \& M. R. Ribeiro (Orgs.), Análise do comportamento: Pesquisa, teoria e aplicação (pp. 1526). Porto Alegre: Artmed.

Lê, A. D., Poulos, C. X., \& Cappel, H. (1979). Conditioned tolerance to the hypothermia effect of ethyl alcohol. Science, 206, 1109-1110.

Lejuez, C. W., Schaal, D. W., \& O’Donnell, J. (1998). Behavioral pharmacology and the treatment of substance abuse. Em J. J. Plaud \& G. H. Eifert (Orgs.), From behavior theory to behavior therapy (pp. 116-135). Boston, MA: Allyn \& Bacon.

Leslie, J. C. (2003). A history of reinforcement: The role of reinforcement schedules in behavior pharmacology. The Behavior Analyst Today, 4, 98-108.

Lovibond, S. H., \& Caddy, G. (1970). Discriminated aversive control in the moderation of alcoholics drinking behavior. $\mathrm{Be}$ havior Therapy, 1, 437-444. 
Lubinski, D., \& Thompson, T. (1987). An animal model of interpersonal communication of interpersonal communication of interoceptive (private) states. Journal of the Experimental Analysis of Behavior, 48, 1-15.

Macrae, J. R., Scoles, M. T., \& Siegel, S. (1987). The contribution of pavlovian conditioning to drug tolerance and dependence. British Journal of Addiction, 82, 371-380.

Marr, M. J. (2006). A major trio. Journal of the Experimental Analysis of Behavior, 86, 355-357.

Miguel, C. F. (2000). O conceito de operação estabelecedora na análise do comportamento. Psicologia: Teoria e Pesquisa, 16, 259-267.

Morris, E. K., Lazo, J. F., \& Smith, N. G. (2004). Whether, when, and why Skinner published on biological participation in behavior. The Behavior Analyst, 27, 153-169.

Northup, J., Fusilier, I., Swanson, V., Roane, H., \& Borrero, J. (1997). An evaluation of methylphenidate as a potential establishing operation for some common classroom reinforcers. Journal of Applied Behavior Analysis, 30, 615-625.

Poling, A. (2000). Introduction. Em A. Poling \& T. Byrne (Orgs.), Introduction to behavioral pharmacology (pp. 9-24). Reno, NV: Context Press.

Poling, A., Byrne, T., \& Morgan, T. (2000). Stimulus properties of drugs. Em A. Poling \& T. Byrne (Orgs.), Introduction to behavioral pharmacology (pp. 141-166). Reno, NV: Context Press.

Reese, H. W. (1996a). How is physiology relevant to behavior analysis? The Behavior Analyst, 19, 61-70.

Reese, H. W. (1996b). Response to commentaries. The Behavior Analyst, 19, 85-88.

Schuster, C. R. (1976). Drugs as reinforcers in monkey and man. Pharmacological Reviews, 27, 511-521.

Siegel, S. (1975). Evidence from rats that morphine tolerance is a learned response. Journal of Comparative and Physiological Psychology, 89, 498-506.

Siegel, S. (2001). Pavlovian conditioning and drug overdose: When tolerance fails. Addiction Research \& Theory, 9, 503513.
Siegel, S., Hinson, R. E., Krank, M. D., \& McCully, J. (1982). Heroin "overdose" death: Contributions of drug-associated environmental cues. Science, 216, 436-437.

Skinner, B. F. (1991). The behavior of organisms: An experimental analysis. Acton, MA: Copley. (Original publicado em 1938)

Skinner, B. F. (1965). Science and human behavior. New York, NY: Free Press. (Original publicado em 1953)

Skinner, B. F. (1992). Verbal behavior. Acton, MA: Copley Publishing Group. (Original publicado em 1957)

Skinner, B. F. (1969). The inside story. Em B. F. Skinner (Org.), Contingencies of reinforcement: A theoretical analysis (pp. 269-297). New York, NY: Appleton-Century-Crofts.

Skinner, B. F. (1976). About behaviorism. New York: Vintage Books. (Original publicado em 1974)

Skinner, B. F. (1990). Can psychology be a science of mind? American Psychologist, 45, 1206-1210.

Strumwasser, F. (1994). The relations between neuroscience and human behavioral science. Journal of the Experimental Analysis of Behavior, 61, 307-317.

Thompson, T. I., \& Schuster, C. R. (1968). Behavioral pharmacology. Englewood Cliffs, NJ: Prentice Hall.

Timberlake, W., Schaal, D. W., \& Steinmetz, J. E. (2005). Relating behavior and neuroscience: Introduction and synopsis. Journal of the Experimental Analysis of Behavior, 84, 305-311.

Todorov, J. C. (2004). Da aplysia à constituição: Evolução de conceitos na análise do comportamento. Psicologia: Reflexão e Crítica, 17, 151-156.

Zeiler, M. D. (2006). An architect of the golden years. Journal of the Experimental Analysis of Behavior, 86, 385-391.
Recebido: 12/01/2011

Última revisão: 23/05/2011 Aceito: 19/07/2011 